\title{
Vortex Configurations on Mesoscopic Cylinders with Square Cross Section
}

\author{
Mauro M. Doria \\ Instituto de Física, Universidade Federal do Rio de Janeiro, \\ C.P. 68528, 21945-970, Rio de Janeiro RJ, Brazil \\ and Gilney Figueira Zebende \\ Departamento de Física, Universidade Estadual de Feira de Santana, \\ Feira de Santana, BA, Brazil \\ Received on 28 February, 2002
}

\begin{abstract}
Very long and thin superconducting cylinders with square cross section are studied using a modified Ginzburg Landau theory that incorporates the boundary conditions into the free energy expression.
\end{abstract}

Recent developments in the nanofabrication technologies brought new interest into the study of mesoscopic superconductors [1, 2]. Consequently theorists went back to discuss many problems that could have been solved long ago if the motivation existed before. The confinement of vortices in samples with sizes of the order of the coherence length produce interesting effects such as the onset of giant vortices and meta-stability, properties not found in pure systems on the bulk. For this reason such small scale superconductors have become of general interest $[3,4,5]$.

We study here a type II superconductor with the shape of a very thin and long cylinder with square cross section. The applied field is parallel to the cylinder and the side of the square is just a few times larger than the coherence length $\xi_{0}$. Because the cylinder is very long the problem becomes two-dimensional and it is enough to study a plane perpendicular to the cylinder. We assume that the London penetration length is much larger than the side of the square, $L$, and consequently, than $\xi_{0}$ too. There is no shielding of the applied field and the magnetic field is constant everywhere, being equal to the applied field. To describe the equilibrium configurations of this system we use a modified Ginzburg-Landau theory which automatically incorporates the appropriate boundary conditions into the theory and presents a unified view of both normal and superconducting regions independently of their shape [6]. The present treatment of the square shape cross section shows that this theory is also able to treat other geometries, although this is not done here. The method treats superconducting and normal regions on equal footing through a step-like function $\tau(\vec{x})$, zero in the normal and one in the superconducting regions, respectively, according to the free energy density expansion below,

$$
\begin{aligned}
F & =(1 / V) \int d v\left\{\tau(\vec{x})|\vec{D} \psi|^{2} / 2 m\right. \\
& \left.+\tau(\vec{x}) \alpha_{0}\left[T-T_{c}\right]|\psi|^{2}+\beta|\psi|^{4} / 2\right\}
\end{aligned}
$$

where $\vec{D} \equiv(\hbar / i) \vec{\nabla}-q \vec{A} / c$, and $\vec{\nabla} \times \vec{A}=\vec{H}$.

The well-known Saint-James-de Gennes [7] boundary condition,

$$
\hat{n} \cdot \vec{D} \psi=0
$$

is automatically satisfied at the normalsuperconducting interfaces by this theory. The solution in the normal region is trivial since Eq.(1) reduces to $F=(1 / V) \int d v\left\{\beta|\psi|^{4} / 2\right\}$, with the trivial solution $\psi=0$.

The solutions of the above modified GinzburgLandau theory in the square geometry are found numerically using a discretized version of the theory. An array of $N^{2}$ points is introduced and consequently the field $\psi(x, y)$ is replaced by $\Delta\left(n_{x}, n_{y}\right), n_{x}=1, \ldots, N$, and $n_{y}=1, \ldots, N$. The discretized version theory is gauge invariant. The numerical method used to search for solutions is the Simulated Annealing method[8]. The Simulated Annealing method is a Monte Carlo thermalization procedure that contains a temperature parameter, which is automatically lowered until a solution that meets a convergence criteria is found. In 
our procedure at least 1600 visits are made at each site for a given Monte Carlo temperature. The system undergoes an average of 150 temperature reductions, though this number depends on how fast the absolute minimum is reached. The new order parameter, $\Delta=\sqrt{\beta / \alpha_{0}\left(T_{c}-T\right)} \psi$ is normalized such that the density, $\left|\Delta\left(n_{x}, n_{y}\right)\right|^{2}$,is bound by one (maximum) and zero (minimum). The distance between two consecutive points in the numerical mesh is called $a$, and physical results are invariant under different choices of this parameter. In order that this discrete theory describes the continuum Ginzburg-Landau theory we must have the coherence length, $\xi(T)=\sqrt{\hbar^{2} / 2 m \alpha_{0}\left(T_{c}-T\right)}, \xi_{0}=$ $\xi(0)$, larger than $a$. We choose to do our simulations for $T=0$ and this is equivalent to fixing the parameters $R / \xi_{0}$ and $\xi_{0} / a_{i}$ to constant values.

The step-like function $\tau(\vec{x})$ that describes the square is,

$$
\tau(\vec{x})=\frac{2}{1+\exp \left[(2|x| / L)^{K}\right]} \frac{2}{1+\exp \left[(2|y| / L)^{K}\right]}
$$

where we take $K=8$.

Although the present theory has no $\kappa$ parameter it is possible to simulate the limit $\kappa \rightarrow \infty$ using the following trick. The magnetization is obtained through the expression,

$$
\vec{M}=(1 / V) \int d v\{\vec{x} \times \vec{J} / 2 c\},
$$

where $\vec{J}=(q / 2 m)[\psi * \vec{D} \psi+c . c]$ is the electromagnetic current. The above expression gives the magnetization just from the spatial distribution of the order parameter. However Ampere's law is not being imposed here and the Meissner regime does not exist in the present study. It happens that the above magnetization is only proportional to the true magnetization of the limit $\kappa \rightarrow \infty$. The proportionality constant, $C$, is determined imposing that $B=H+C \cdot 4 \pi M=0$ for very small $H$ values.

Let us discuss the results of our numerical minimization of the free energy, described by Eq.(1), whose results are shown in the figures.

Figure 1 shows two curves on a single plot, namely the free energy versus the applied field and the magnetization versus the applied field for a square with side $L=2 \xi_{0}$. In the small applied field regime the magnetization is approximately linear and this is associated to the Meissner phase. For increasing field the magnetization reaches a maximum after the Meissner region and then decreases finally disappearing for a maximum applied field. No vortices enter the superconductor for $L=2 \xi_{0}$. These two curves are also shown in Fig. 2 for a square with side $L=3 \xi_{0}$. In this case there is a discontinuity in the magnetization as the applied field changes from $H_{A}$ to $H_{B}$. At this point the free energy undergoes a slope discontinuity indicating a first order transition. It corresponds to the entrance of the first vortex inside the square. Thus we have just determined here that the critical side of square where the entrance of a vortex becomes possible should be between $2 \xi_{0}$ and $3 \xi_{0}$. Fig. 3 shows the free enrgy and magnetization curves for a larger square, that is, of side $L=8 \xi_{0}$. Several transitions are observed in this case, each occuring at special values of the applied field. From zero field to $H_{A}$ there are no vortices inside the system. $H_{B}$ marks the entrance of the first vortex, $H_{C}$ of the second, $H_{D}$ of the third, and $H_{E}$ of the fourth. For fields larger than $H_{E}$ more vortices enter the square, although a more accurate curve than the present one is necessary to characterize these transitions. We have observed that individual vortices tend to collapse into a single giant vortex as the field increases, until a new vortex enters when they again are individually seen. For instance, the field value imediately before $H_{E}$ corresponds to just a single giant vortex with vorticity three. Next we show the normalized order parameter for each point of our mesh, taken with $N=60$ and lattice parameter $a=\xi_{0} / 5$. Figs. 4, 5, 6, 7, 8 correspond to the applied field values $H_{A}, H_{B}, H_{C}$, and $H_{D}$, respectively. Each depression of the order parameter correspond to a vortex inside the system.

In conclusion we have studied here a long superconducitng cylinder with a square cross section using a modified Ginzburg-Landau theory that naturally incorporates the appropriate boundary conditions of normalsuperconducting interfaces. We numerically solve a discrete version of this theory using the Simulated Annealing method. We showed that this method gives a good description of the present square geometry. The entrance of vortices produce jumps in the magnetization showing that they induce first order transitions in the system, as shown for the cases of squares with sides $L=2 \xi_{0}, L=3 \xi_{0}$, and $L=8 \xi_{0}$ studied here.

\section{Acknowledgments}

This work was supported in part by $\mathrm{CNPq}$ and FAPERJ. 


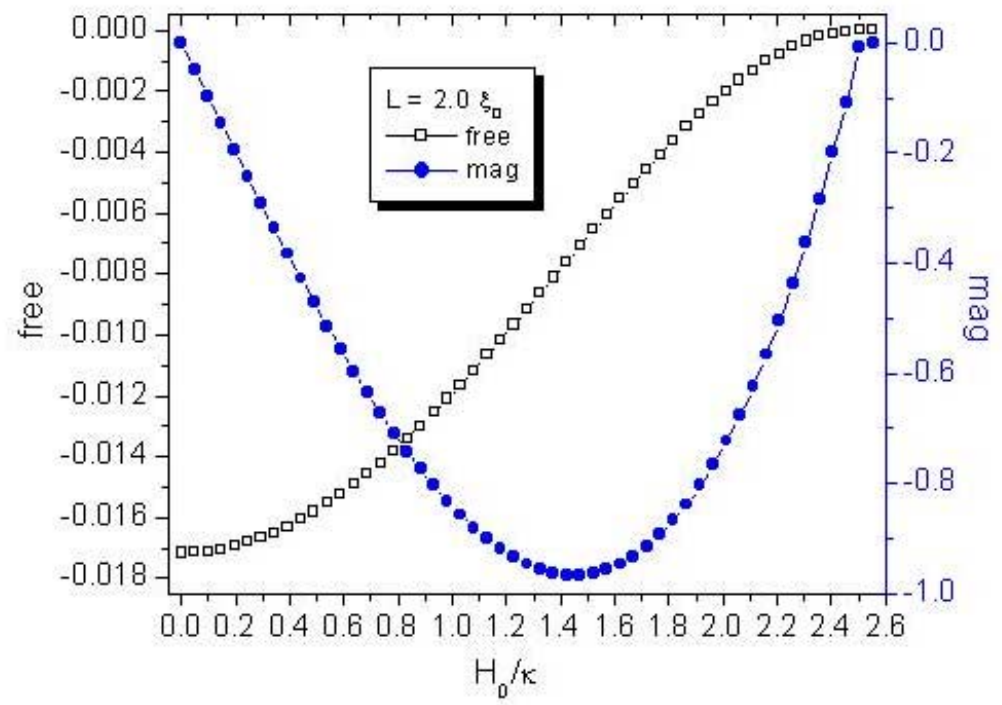

Figure 1. The free energy and the magnetization versus the applied field are shown here for the case of a square with side $L=2 \xi_{0}$. The magnetization has no discontinuities indicating that no vortices penetrate in the system.

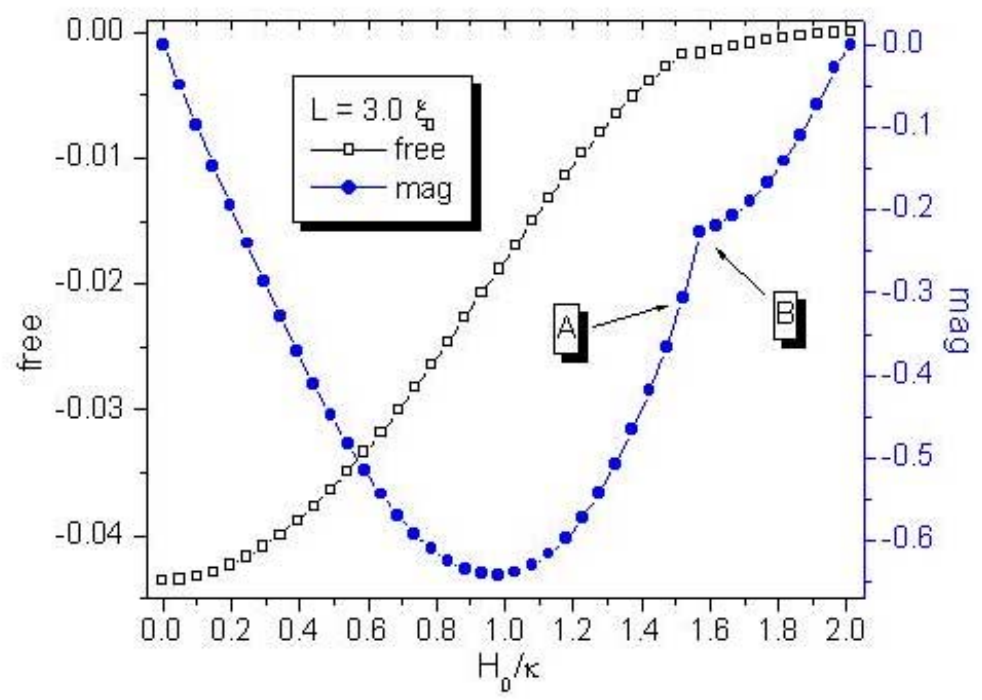

Figure 2. The free energy and the magnetization versus the applied field are shown here for the case of a square with side $L=3 \xi_{0}$. The magnetization undergoes a jump from A to B indicating that a vortex has penetrated into the system. 


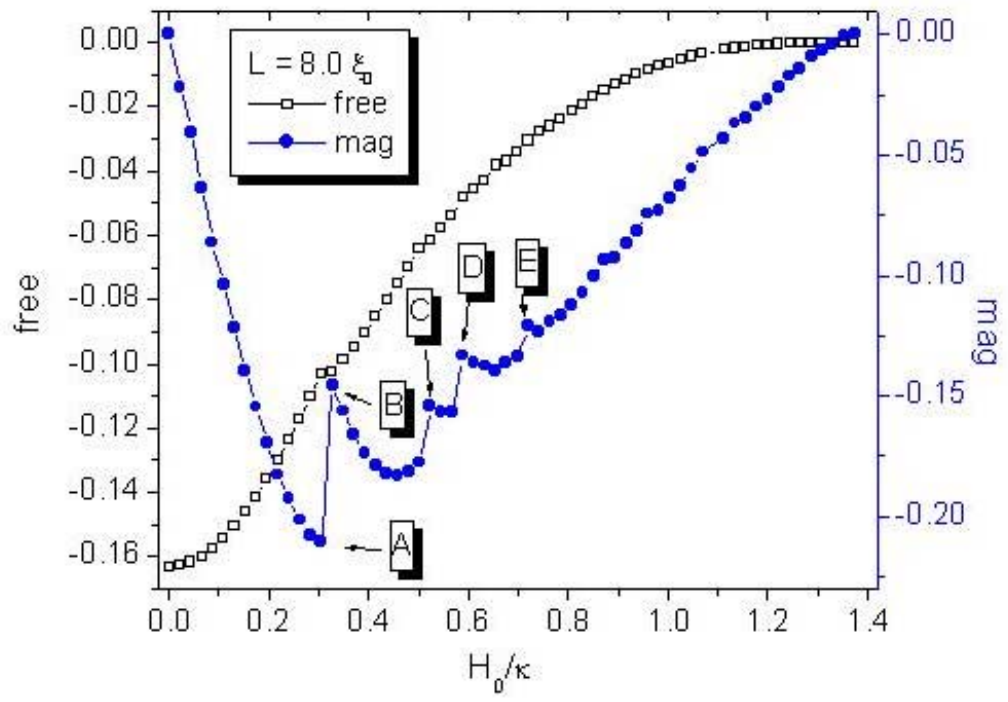

Figure 3. The free energy and the magnetization versus the applied field are shown here for the case of a square with side $L=8 \xi_{0}$. The jumps in the magnetization characterize entrance fields for vortices. From no field until $H_{A}$ there is no vortex inside the system and at $H_{B}$ a vortex enters the system. The other fields are associated with new number of vortices inside the system, namely $H_{C}$ two vortices, $H_{D}$ three, and $H_{E}$ four.

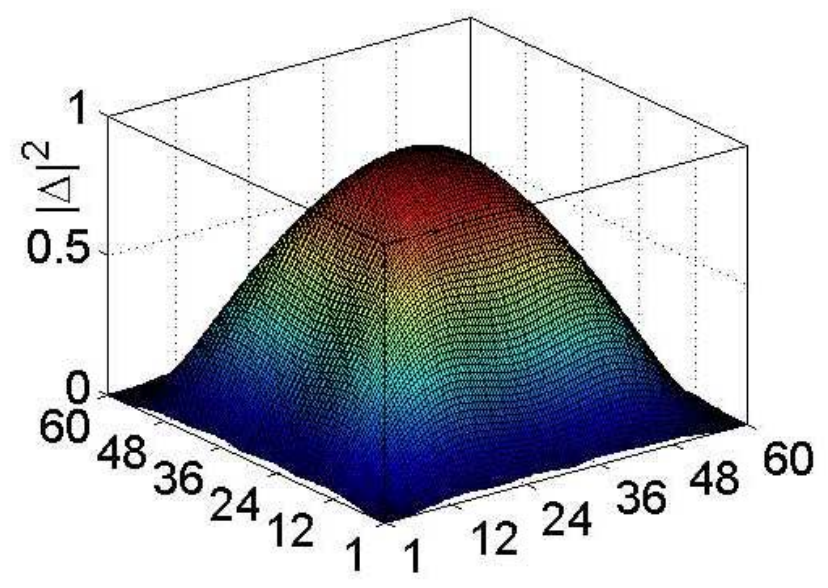

Figure 4. The normalized order parameter $\mid \Delta\left(n x,\left.n y\right|^{2}\right.$ is shown here for $L=8 \xi_{0}$ and field value $H_{A}$.

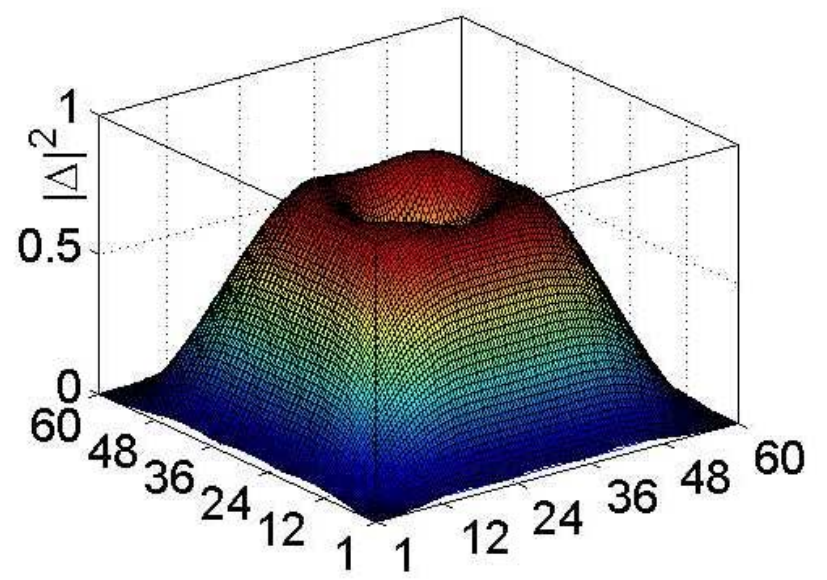

Figure 5. The normalized order parameter $\mid \Delta\left(n x,\left.n y\right|^{2}\right.$ is shown here for $L=8 \xi_{0}$ and field value $H_{B}$. 


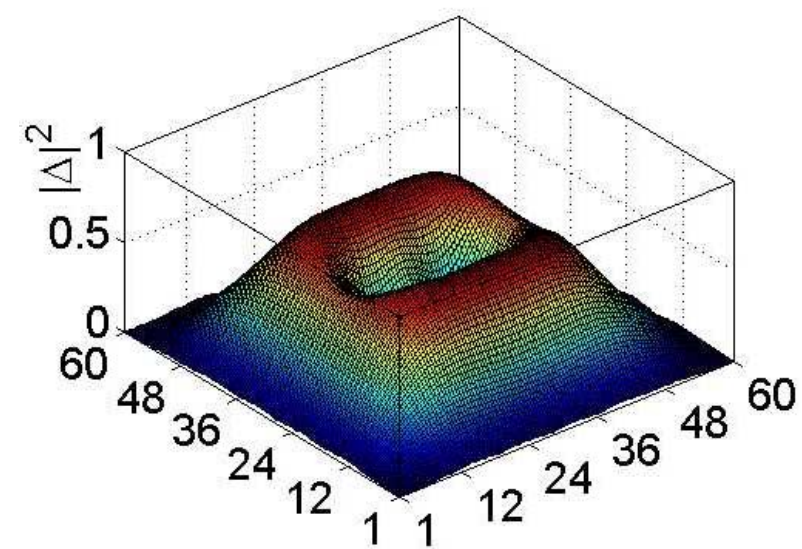

Figure 6. The normalized order parameter $\mid \Delta\left(n x,\left.n y\right|^{2}\right.$ is shown here for $L=8 \xi_{0}$ and field value $H_{C}$.

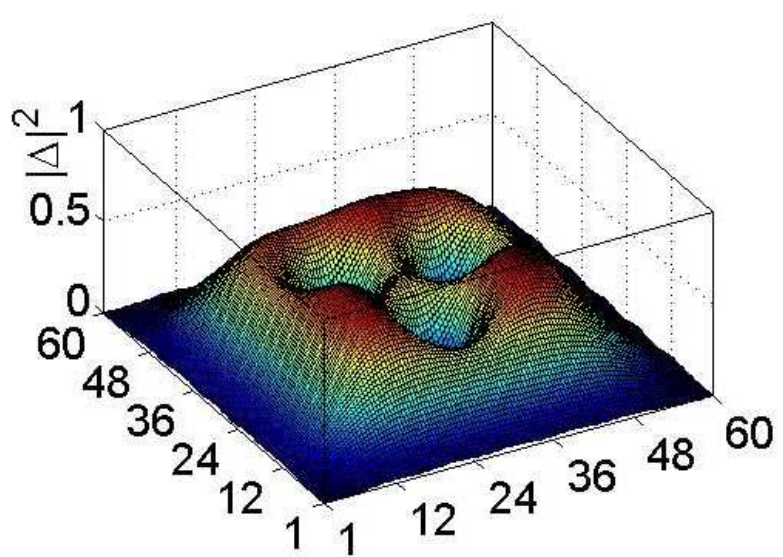

Figure 7. The normalized order parameter $\mid \Delta\left(n x,\left.n y\right|^{2}\right.$ is shown here for $L=8 \xi_{0}$ and field value $H_{D}$.

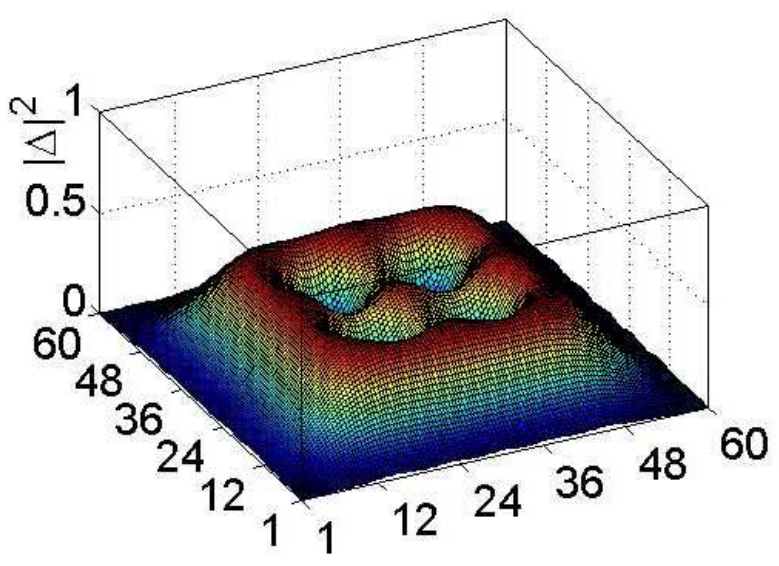

Figure 8. The normalized order parameter $\mid \Delta\left(n x,\left.n y\right|^{2}\right.$ is shown here for $L=8 \xi_{0}$ and field value $H_{E}$.

\section{References}

[1] O. Buisson, P. Gandit, R. Rammel, Y.Y. Wang and B. Pannetier, Phys. Lett. A 66, 15036.

[2] A.K. Geim, I.V. Grigorieva, S.V. Dubonos, J.G.S. Lok, J.C. Maan, A.E. Filippov and F.M. Peeters, Nature 390, 259 (1997).

[3] J.J. Palacios, Phys. Rev. Lett. 84, 1796 (2000).

[4] B.J. Baelus, F.M. Peeters and V.A. Schweigert, Phys. Rev. B 63, 144517 (2001).

[5] S.Y. Yampolskii and F.M. Peeters, Phys. Rev. B 62, 9663 (2000).

[6] Mauro M. Doria and Sarah C.B. de Andrade, Phys. Rev. B 60, 13164 (1999).

[7] D. Saint-James and P.G. de Gennes, Phys. Lett. 7, 306 (1963).

[8] M.M. Doria and J.E. Gubernatis and D. Rainer, Phys. Rev. B 41, 6335 (1990). 